\title{
Experiments with a High-Density Positronium Gas
}

\author{
D. B. Cassidy, ${ }^{1}$ S. H. M. Deng, ${ }^{1}$ R. G. Greaves,${ }^{2}$ T. Maruo,${ }^{3}$ N. Nishiyama, ${ }^{3}$ J. B. Snyder, ${ }^{4}$ \\ H. K. M. Tanaka, ${ }^{1}$ and A.P. Mills, Jr. ${ }^{1}$ \\ ${ }^{1}$ Department of Physics, University of California, Riverside, California 92521-0413, USA \\ ${ }^{2}$ First Point Scientific Inc., Agoura Hills, California 91301, USA \\ ${ }^{3}$ Division of Chemical Engineering, Osaka University, 1-3 Machikaneyama, Toyonaka, Osaka 560 8531, Japan \\ ${ }^{4}$ Department of Physics, Principia College, Elsah, Illinois 62028, USA \\ (Received 4 October 2005; published 4 November 2005)
}

\begin{abstract}
We have created a high-density gas of interacting positronium (Ps) atoms by irradiating a thin film of nanoporous silica with intense positron bursts and measured the Ps lifetime using a new single-shot technique. When the positrons were compressed to $3.3 \times 10^{10} \mathrm{~cm}^{-2}$, the apparent intensity of the orthopositronium lifetime component was found to decrease by $33 \%$. We believe this is due to a combination of spin exchange quenching and $\mathrm{Ps}_{2}$ molecule formation associated with colliding pairs of oppositely polarized triplet positronium atoms. Our data imply an effective cross section for this process of $2.9 \times 10^{-14} \mathrm{~cm}^{-2}$.
\end{abstract}

DOI: 10.1103/PhysRevLett.95.195006

PACS numbers: 52.27.Jt, 36.10.Dr, 34.80.Nz

Developments in positron trapping and accumulation technology in recent years [1] have opened the door to a number of new experimental regimes, making feasible some hitherto impossible experiments. For example, the use of positron plasmas was a key factor in the success of the first attempts to produce cold antihydrogen [2], and high quality positron beams derived from trapped plasmas have been used to perform detailed studies of positron interactions with atoms and molecules [3].

Another area that is expected to benefit from similar methods is that of many positronium systems [4], of which there have been no previous experimental studies at all. Indeed, even though the positron was first observed in 1933 [5], positronium in 1951 [6], and the positronium negative ion in 1981 [7], the positronium positive ion and the positronium molecule (which are the next members of the polyelectron series envisioned by Wheeler [8]) have not yet been observed, because the required high positron densities were not available. By accumulating many positrons in a Surko-type trap [1] and performing certain manipulations upon the resulting plasma, we have been able to generate positron bursts with spatiotemporal densities sufficiently high to allow us to observe interactions between positronium atoms.

The experiment was performed using a positron trap based on that described in Ref. [9]. A slow positron beam was generated using a $20 \mathrm{mCi}{ }^{22} \mathrm{Na}$ source and a solid neon moderator, producing a beam of $(6 \pm 1) \times$ $10^{6} e^{+} \mathrm{s}^{-1}$, which was then injected into a two stage Surko-type trap [1]. The output from this trap was sent to an accumulator (as shown in Fig. 1), where many positron pulses were stacked, and compressed radially with a rotating wall electric field [10], producing long-lived plasmas $(\tau>1000 \mathrm{sec})$ containing up to $100 \times 10^{6}$ positrons in a $500 \mathrm{G}$ axial magnetic field.
The accumulator output was focused in time by rapidly applying a potential gradient [shown in Fig. 1(b)] across the extent of the accumulator electrodes [11] leading to positron pulses with a duration of 15-20 ns (FWHM). Data were recorded using a lead tungstate $\left(\mathrm{PbWO}_{4}\right)$ scintillator attached to an XP2020 photomultiplier. This scintillator material was chosen because it has a relatively fast decay time $(<15 \mathrm{~ns})$ and a high density $\left(8.9 \mathrm{~g} / \mathrm{cm}^{3}\right)$. The anode signal from the detector was connected directly to an Agilent 54885A digital storage oscilloscope.

The target material used in this work was a thin $(\sim 450 \mathrm{~nm})$ film of porous silica prepared on a silicon
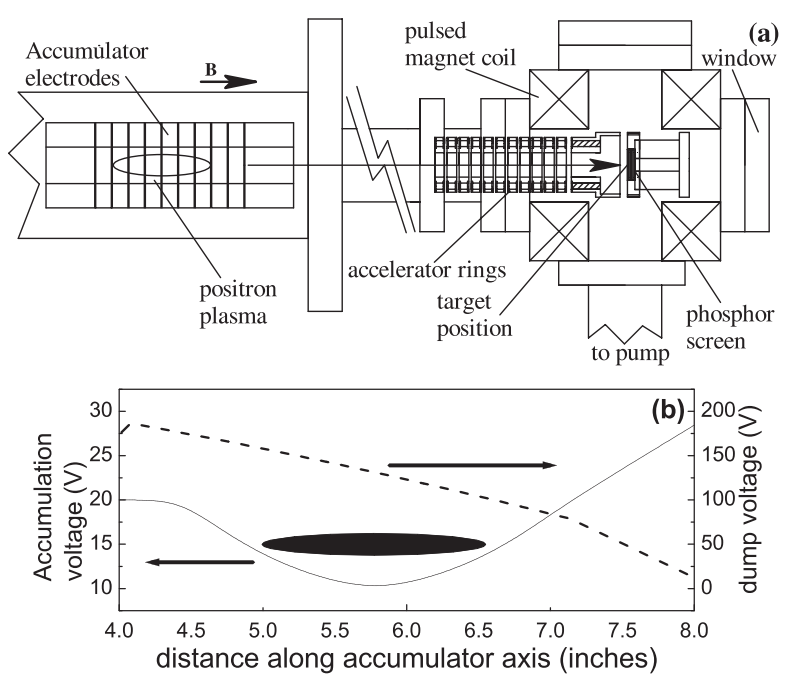

FIG. 1. (a) Schematic layout of the accumulator and target chamber. When the target was retracted, the beam could be imaged using the phosphor screen and a CCD camera looking through the window. The distance from the accumulator to the target was $1 \mathrm{~m}$. (b) Potential structure in the accumulator for filling and dumping. 
wafer substrate [12]. Positrons injected into this type of nanoporous material form positronium in the bulk with a relatively high efficiency, and positronium lifetime spectroscopy is a well-established technique that is often used to characterize many of the different properties of similar materials [13].

Ground state positronium is formed with equal likelihood in each of the three triplet states (lifetime $142 \mathrm{~ns}$ ) and in the singlet state (lifetime $125 \mathrm{ps)} \mathrm{[14].} \mathrm{If} \mathrm{the} \mathrm{positrons}$ from which the positronium is formed are not perfectly spin polarized, there may be spin-exchanging triplet Pstriplet Ps collisions that will increase the average annihilation rate [15]. Spin exchange quenching of two triplet Ps atoms approaching each other at low relative momenta can occur only when the two atoms have total $S=0$, in which case the state has the same quantum numbers as the state with two singlet atoms. The most dramatic result of such a collision is an increase in the decay rate that is proportional to the density; this is the basis of our measurement.

In the present experiment, $\sim 15 \times 10^{6}$ positrons were dumped onto the target in a single pulse about $\sim 20 \mathrm{~ns}$ wide. The large number of positrons in each pulse clearly precludes the use of a conventional positron annihilation lifetime spectroscopy (PALS) system, which is based on counting single photons [16]. Thus, we have developed a new technique for single-shot positron annihilation lifetime spectroscopy (SSPALS), wherein the detector anode is connected to a fast oscilloscope which is triggered by the accumulator dump signal and records an entire lifetime spectrum in a single shot. This arrangement has a timing resolution that depends on the decay time of the detector (in this case, $15 \mathrm{~ns}$ ) and is, therefore, much lower than that of conventional PALS. However, for the purposes of measuring orthopositronium lifetimes that are of the order of the vacuum lifetime, SSPALS is more than adequate. Besides being a tool for high-density Ps experiments, the SSPALS technique introduced here has the potential for other applications. For example, it could be used for studying time dependent defect densities in surfaces irradiated by intense laser pulses.

Figure 2 shows a set of three lifetime curves, each obtained using a single pulse of $1.4 \times 10^{7}$ positrons with a full width at half maximum time spread of $20 \mathrm{~ns}$. The positrons are compressed using the rotating wall effect and may then be expanded by switching off the rotating wall for a variable expansion time $t_{x}$ of $0-5 \mathrm{sec}$ before being ejected. The positron pulse is subsequently compressed in a pulsed $\sim 1 \mathrm{~T}$ magnetic field to a beam of Gaussian cross section having a minimum ("compressed") full width at half maximum diameter of $0.20 \pm 0.02 \mathrm{~mm}$ corresponding to a central areal density of $3.3 \times 10^{10}$ positrons per $\mathrm{cm}^{2}$.

One of the lifetime spectra shown in Fig. 2 is taken with a positron energy of $7 \mathrm{keV}$ using an uncleaned Si target, for which no Ps exists in the bulk, and less than $2 \%$ of the positrons form Ps in vacuum from surface processes. Two spectra were obtained, with the beam expanded and compressed (see inset). These spectra were indistinguishable,

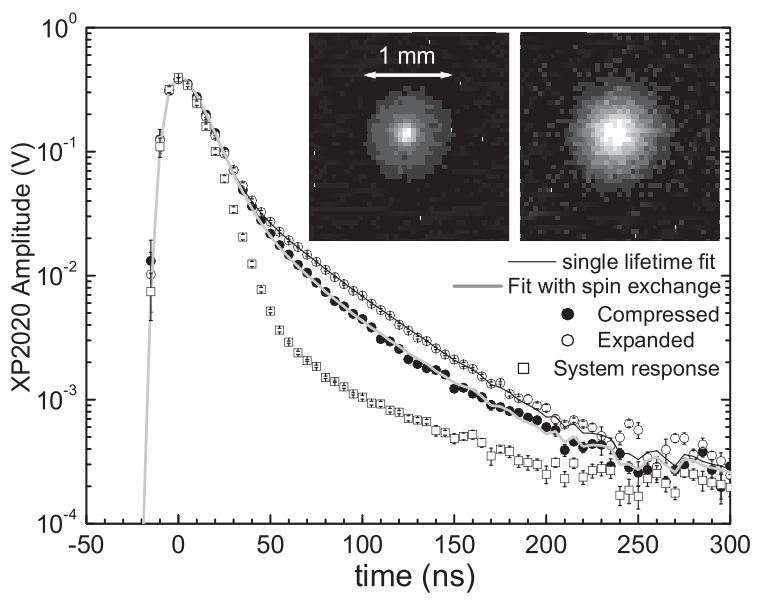

FIG. 2. Single-shot lifetime spectra for compressed and expanded beams as well as the system resolution data taken using a silicon target. The solid lines are fits including the system response function (see text). The inset shows the beam profiles for a compressed and an expanded beam, with areal densities of 3.3 and $0.49 \times 10^{10} \mathrm{~cm}^{-2}$, respectively.

indicating that there is no observable effect due to high positron densities in the absence of Ps formation. The average of the two spectra was taken to be the instrumental response function, and we have ignored the effect of the $<2 \%$ vacuum Ps component on our fits.

In the remaining two spectra, positrons were implanted with an energy of $3 \mathrm{keV}$ into the porous silica sample. The $|m|=1$ o-Ps lifetime measured using the expanded beam was fitted by a single component with decay time of $36.4 \pm$ $0.1 \mathrm{~ns}$ with an intensity $I_{\mathrm{oPs}|m|=1}=24.3 \pm 0.2 \%$. The lifetime data obtained using a compressed pulse cannot be fitted using a single lifetime decay and require a more detailed model.

Because of the high magnetic field at the target (1 T), the $m=0$ triplet lifetime is quenched [14] to less than $7 \mathrm{~ns}$, which is much less than our time resolution; the $m= \pm 1$ components are unaffected by the magnetic field. At low densities, the $|m|=1$ o-Ps decays at a rate $\gamma$ that is the sum of the self-annihilation rate into three photons and the pickoff annihilation rate (predominately into two photons) due to collisions of the o-Ps with electrons of the surrounding medium. At high positronium densities, there will be spin-exchanging collisions between pairs of oppositely polarized $|m|=1$ o-Ps atoms. So long as the Ps kinetic energy is small compared to the binding energy $E_{M}=$ $0.44 \mathrm{eV}$ of the $\mathrm{Ps}_{2}$ molecule [17], spin exchange will be determined by the singlet channel (total spin zero) Ps-Ps scattering length $a_{0}=0.444 \mathrm{~nm}$, with total cross section $\sigma_{0}=4 \pi a_{0}^{2} \approx 2.48 \times 10^{-14} \mathrm{~cm}^{2}[18] .|m|=1$ Ps atoms could lead to the formation of $\mathrm{Ps}_{2}$ molecules. This will occur at a rate dependent on the available surface area of the sample, since wall interactions are needed to conserve energy and momentum in the absence of three body collisions. Let $n_{ \pm}(\vec{x}, t)$ be the number per unit volume at position $\vec{x}$ and time $t$ of orthopositronium atoms with 
magnetic quantum number $m= \pm 1$. The rate of disappearance of $m= \pm 1$ atoms due to collisions with $m=\mp 1$ atoms will then be

$$
d \ln n_{ \pm}(\vec{x}, t) / d t=n_{\mp}(\vec{x}, t) \sigma_{T} \sqrt{2} \bar{\nu},
$$

where $\sigma_{T}=\sigma_{x}+\sigma_{W}$ and $\bar{\nu} \approx 7.6 \times 10^{6} \mathrm{~cm} / \mathrm{s}$ is the mean velocity of a Ps atom at room temperature (the incident beam will cause negligible target heating). Here $\sigma_{x}=\sigma_{0} / 3=0.654 \times 10^{-14} \mathrm{~cm}^{2}$ is the spin exchange cross section at low velocity for collisions of a triplet $m=$ \pm 1 with an $m=\mp 1$ atom in which the result is either a p-Ps or o-Ps atom with $m=0$, both of which will rapidly annihilate. The effective cross section for forming $\mathrm{Ps}_{2}$ molecules at the walls of the pores is $\sigma_{W}$, which will depend in part on an effective interaction length that includes effects due to the Ps wave function in the pore, coupling to phonons of the silica matrix, etc.

The rate equations for Ps decay including a Gaussian beam profile and an assumed positron polarization of $35 \%$ are used to obtain the fitted curve for the compressed beam data in Fig. 2. The fitted curve implies $\sigma_{T}=2.9 \times$ $10^{-14} \mathrm{~cm}^{2}$, assuming the porosity of the sample is 0.70 , the distance over which the positronium is spread out is equal to the sample thickness $(450 \mathrm{~nm})$, and the central areal density of the positron pulse is $n_{2 \mathrm{D}}=3.3 \times$ $10^{10} \mathrm{~cm}^{-2}$. This value of $\sigma_{T}$ is over 4 times higher than would be expected due to spin exchange alone. This may indicate that cracks, voids, or regions of higher porosity in the sample lead to local regions of higher density positronium where the quenching rate increases significantly. However, the fact that $\sigma_{T} \approx 4 \sigma_{x}$ could also mean that there is a significant amount of $\mathrm{Ps}_{2}$ formation on the pore walls. Our present experiment cannot distinguish between spin exchange quenching and $\mathrm{Ps}_{2}$ formation, since they both lead to the same signal (a reduction in the long-lived lifetime component). Studies using a variety of samples with differing internal surface areas and pore sizes should help to clarify this matter.

We have also measured the quenching effect as a function of beam energy. Since the positron implantation profile extends further into the sample as the beam energy is increased, the positronium density and, hence, the quenching rate will also vary with the beam energy. Figure 3 shows an energy scan for the same sample of Fig. 2. The delayed fraction is the ratio of the integral of a lifetime spectrum from $100-300 \mathrm{~ns}$ and from -100 to $300 \mathrm{~ns}$ and is a measure of the long-lived positronium fraction.

An intriguing feature in this data is the increased quenching at very low positron energies, for which the positrons are expected to penetrate only a small distance into the sample. We explain this in the following way: For positron energies around $1 \mathrm{keV}$, positronium is formed close to the sample surface and is, therefore, able to diffuse into the vacuum. For higher energies, the implanted positrons are spread out, with a significant fraction penetrating into the silicon substrate. In both cases, the positronium

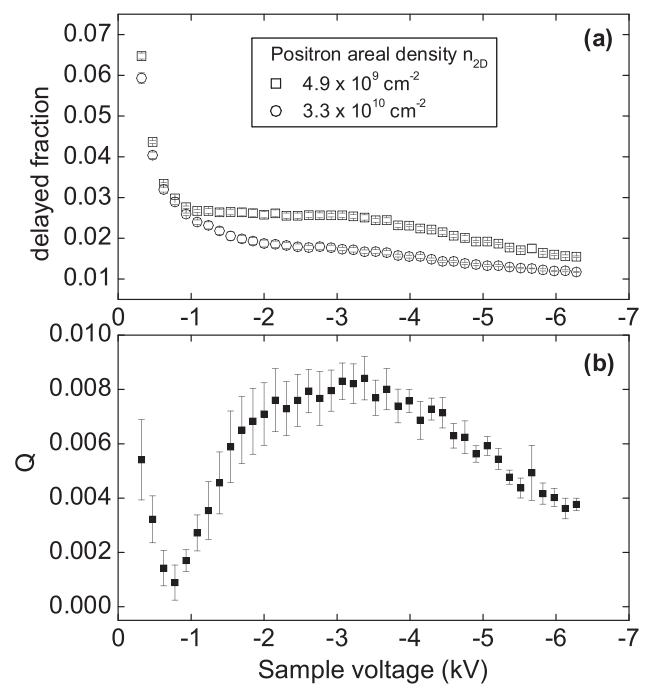

FIG. 3. Delayed fraction as a function of beam energy; (a) is the delayed fraction for an expanded and a compressed beam; (b) shows the quenching signal $Q$, which is the difference between the two.

density and, hence, the quenching effect are reduced, thus explaining the existence of a maximum quenching signal at a positron energy of $\sim 3 \mathrm{keV}$. On the other hand, at very low energies $(<1 \mathrm{keV})$ many epithermal positrons reach the surface and form Ps with an efficiency greater than that with which it is formed in the interior of the sample [see Fig. 3(a)]. Some of this Ps is directly emitted into the vacuum, and the remainder is trapped on the sample surface [19]. At low densities and at room temperature, a portion of these eventually leave the surface due to thermal desorption, while the rest decay with a few nanosecond lifetime by pickoff annihilation with surface electrons. At high densities, the amount of thermally desorbed Ps may diminish due to the production of $\mathrm{Ps}_{2}$ molecules that are emitted into the vacuum or from surface Ps-Ps spin exchange quenching, leading to an increase in the quenching signal. Unfortunately, the present time width of the beam precludes us from studying this effect in any further detail. Work is now underway to produce subnanosecond positron bursts and then obtain conclusive evidence for $\mathrm{Ps}_{2}$ production.

Figure 4 shows the quenching signal as a function of density obtained by sweeping the expansion time $t_{x}$ from 0 to $5 \mathrm{sec}$ while implanting the positrons at $3 \mathrm{kV}$. The density was measured in a separate experiment in which the positron beam was imaged using a phosphor screen (see inset in Fig. 2). The expected linear density dependence of the quenching rate [see Eq. (1)] is clearly observed in Fig. 4.

We have attributed the change in the positronium decay rate with density to spin exchange quenching and/or $\mathrm{Ps}_{2}$ formation, and we now consider possible alternative explanations for our data. One model for positronium formation is the Mogensen spur model [20] in which a positron, after slowing down, interacts with its terminal ionization cloud. If positronium atoms were interacting 


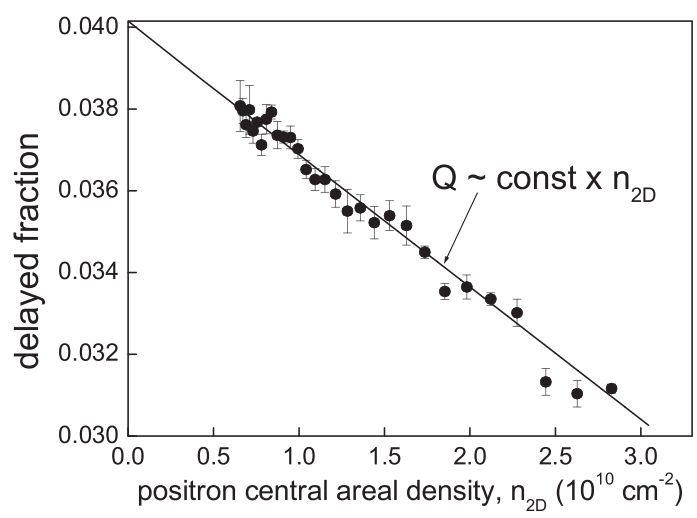

FIG. 4. Density dependence of the quenching effect, $Q=$ counts delayed by more than 100 ns divided by the total counts in the lifetime spectrum.

with such clouds created by different positrons, we might expect a decrease in the lifetime caused by interactions with free radicals and an increase in the apparent intensity due to the availability of free electrons for positronium formation. However, in Fig. 2 the long time curves are essentially parallel, meaning that the lifetime is unchanged by high densities, and the effect at high positron densities is to decrease the apparent Ps intensity. Furthermore, we do not believe alien-spur interactions to be significant, since the spur lifetime is typically around $10 \mathrm{ps}$ while the positron pulse is 2000 times longer, and the spur radius is of the order of $10 \mu \mathrm{m}$, which means that the probability of a positron-alien-spur interaction is less than $10^{-3}$.

To summarize, the synthesis of a number of well known techniques for positron accumulation, brightness enhancement, time bunching, and focusing have led to the highest intensity (cold) positron pulses ever created. The implantation of these pulses into a porous silica material has allowed us to create a gas of interacting positronium atoms, and, using the new technique of SSPALS, we have been able to measure single-shot lifetime spectra from our intense positronium gas bursts and have observed a density dependence in the o-Ps annihilation rate. This constitutes the first observation of spin exchange quenching between pairs of positronium atoms or $\mathrm{Ps}_{2}$ formation. Although we cannot distinguish between these processes, a simple consideration of their rates leads us to believe that we have probably observed some combination of them both.

The areal density we have achieved is thought to be sufficient for a definitive observation of $\mathrm{Ps}_{2}$ molecules [4], and it remains only to increase the temporal compression of the positron pulses and use a metal target (for which there is no Ps surface state) to achieve this goal. Furthermore, this result is an encouraging step towards the realization of the Ps Bose-Einstein condensate (BEC) [15]. A Ps density of $10^{18} \mathrm{~cm}^{-3}$ (3 orders of magnitude greater than the present case) would lead to a BEC transition temperature of $15 \mathrm{~K}$. This could be obtained by a combination of improved rotating wall compression [21], remoderation brightness enhancement [22], and novel porous targets engineered to concentrate the Ps atoms after formation through diffusion into a small cavity.

This work was supported in part by the NSF under Grants No. DMR 0216927 and No. PHY 0140382.

[1] C. M. Surko and R. G. Greaves, Phys. Plasmas 11, 2333 (2004).

[2] M. Amoretti et al., Nature (London) 419, 456 (2002); G. Gabrielse et al., Phys. Rev. Lett. 89, 213401 (2002).

[3] C. M. Surko, G. F. Gribakin, and S. J. Buckman, J. Phys. B 38, R57 (2005).

[4] A. P. Mills, Jr., Nucl. Instrum. Methods Phys. Res., Sect. B 192, 107 (2002).

[5] C. D. Anderson, Phys. Rev. 43, 491 (1933).

[6] M. Deutsch, Phys. Rev. 82, 455 (1951).

[7] A. P. Mills, Jr., Phys. Rev. Lett. 46, 717 (1981).

[8] J. A. Wheeler, Ann. N.Y. Acad. Sci. 48, 221 (1946).

[9] R. G. Greaves and J. Moxom, in Non-Neutral Plasma Physics V, edited by M. Schauer, T. Mitchell, and R. Nebel, AIP Conf. Proc. No. 692 (AIP, New York, 2003), p. 140.

[10] F. Anderegg, E. M. Hollmann, and C.F. Driscoll, Phys. Rev. Lett. 81, 4875 (1998); R. G. Greaves and C.M. Surko, Phys. Rev. Lett. 85, 1883 (2000).

[11] A. P. Mills, Jr., Appl. Phys. (Berlin) 22, 273 (1980).

[12] For details on the particular methods used to prepare the samples, see S. Tanaka, N. Nishiyama, Y. Oku, Y. Egashira, and K. Ueyama, J. Am. Chem. Soc. 126, 4854 (2004).

[13] See, e.g., D. W. Gidley, K. G. Lynn, M.P. Petkov, J. N. Sun, M. H. Weber, and A.F. Yee, in New Directions in Antimatter Chemistry and Physics, edited by D. M. Surko and F. A. Gianturco (Kluwer, Dordrecht, 2001), p. 151.

[14] S. Berko and H. N. Pendleton, Annu. Rev. Nucl. Part. Sci. 30, 543 (1980); A. Rich, Rev. Mod. Phys. 53, 127 (1981).

[15] P. M. Platzman and A. P. Mills, Jr., Phys. Rev. B 49, 454 (1994).

[16] See, e.g., I. K. Mackenzie, in Positron Solid State Physics, Proceedings of the International School of Physics "Enrico Fermi," Course 83, edited by W. Brandt and A. Dupasquier (North-Holland, Amsterdam, 1983), p. 196.

[17] E. A. Hylleraas and A. Ore, Phys. Rev. 71, 493 (1947); Y. K. Ho, Phys. Rev. A 33, 3584 (1986); A. M. Frolov and V. J. Smith, Phys. Rev. A 55, 2662 (1997).

[18] I. A. Ivanov, J. Mitroy, and K. Varga, Phys. Rev. Lett. 87, 063201 (2001).

[19] P. Sferlazzo, S. Berko, and K. F. Canter, Phys. Rev. B 32, 6067 (1985).

[20] O. E. Mogensen, J. Chem. Phys. 60, 998 (1974).

[21] J.R. Danielson and C.M. Surko, Phys. Rev. Lett. 94, 035001 (2005); L. V. Jørgensen et al., Phys. Rev. Lett. 95, 025002 (2005).

[22] A. P. Mills, Jr., Appl. Phys. (Berlin) 23, 189 (1980). 